Sädhanā, Vol. 19, Part 5, October 1994, pp. 817-832. (C) Printed in India.

\title{
Computational study of transport processes in a single-screw extruder for non-Newtonian chemically reactive materials
}

\author{
S GOPALAKRISHNA and Y JALURIA
}

Department of Mechanical and Aerospace Engineering, Rutgers University, New Brunswick, NJ 08903, USA

\begin{abstract}
A numerical study of the transport phenomena arising in a single-screw extruder channel is carried out. A non-Newtonian fluid is considered, using a power law model for the variable viscosity. Chemical reaction kinetics are also included. Finite difference computations are carried out to solve the governing set of partial differential equations for the velocity, temperature and species concentration fields, over a wide range of governing parameters for the case of a tapered screw channel.

The numerical treatment for this combined heat and mass transfer problem is outlined. A marching procedure in the down-channel direction is adopted and the validity of the scheme for practical problems discussed. For large viscous dissipation, the material heats up considerably due to the prevailing shear field, affecting the viscosity significantly, and results in large changes in the pressure development at the end of the channel. The rate of reaction controls the mass diffusion rate which in turn affects viscosity and the flow significantly. The dimensionless throughput, $q_{v}$, is one of the most important parameters in the numerical solution. The dimensionless pressure variation is very sensitive to $q_{v}$, and orders of magnitude changes are possible for small variations in $q_{v}$. Schemes for dealing with other important effects such as back flow, heat transfer by conduction in the barrel, and the effect of the die are also outlined.
\end{abstract}

Keywords. Transport phenomena; single-screw extruder; chemical reaction kinetics; finite difference computations; non-Newtonian materials.

\section{Introduction}

Screw extrusion is a thermomechanical processing operation in which the raw material is fed into a hopper and forced through the passage between a rotating screw and a stationary barrel. The processed material comes out through a die of a specific shape. Single- and twin-screw extruders are used widely in the food and plastics processing industry for the production of shaped and cooked products. The high shear and temperature environment inside the screw channel results in mixing of the material

A list of symbols is given at the end of the paper 
and leads to chemical reactions that constitute the cooking process. The underlying heat and mass transport processes have been analysed, but a comprehensive treatment that is useful for extruder design and optimization is not available. This chapter attempts to fill the gap in the literature by numerically simulating the complex heat and mass transfer interactions for the simple geometry of the single-screw extruder.

Several researchers have studied the flow of polymers in the various sections of an extruder, using different numerical or analytical techniques (Fenner 1977, 1979; Tadmor \& Gogos 1979). Fenner (1977) considered the case of the temperature profile developing along the length of the screw channel. Elbirli \& Lindt (1984) have reported the results from a model in which the temperature was allowed to develop along the screw channel. In these models, the screw and the barrel were assumed to be at the same uniform temperature. Karwe \& Jaluria (1990) have presented numerical results for flow and heat transfer for polymeric materials in single-screw extruders with an adiabatic boundary condition at the screw. There is no available literature on the simulation of mass transport in screw extrusion, even though it is of primary practical importance in terms of product quality and attributes of the extrudate.

A finite difference numerical study has been carried out here for the simulation of flow and transport processes in extrusion of food and plastic materials, to obtain the velocity, temperature, and mass concentration variation along the length of the screw channel. Starch granules are gelatinized by means of water absorption inside the extruder. This conversion process is modelled in our analysis. The variation in channel depth due to taper is accounted for in the model. The effects of viscous dissipation and chemical reaction are included, and the results obtained are discussed in terms of the basic transport processes. The main governing dimensionless parameters are the taper angle $\psi$, the throughput, or volume flow rate $q_{v}$, the Peclet number Pe, which represents the relative importance of convection along the screw channel as compared to conduction, the power law index $n$, the dimensionless viscosity coefficients $\beta, \beta_{1}$, and $b_{m}$, which characterize the variation of viscosity with temperature and concentration, the dimensionless gelatinization rate $S$, and the Griffith number $G$, which represents the level of viscous dissi jation compared to heat conduction. The analysis applies to the metering section or the melt conveying section, where the material is heated and subjected to high shear rates. The effect of the governing parameters on the flow, heat transfer, and concentration distribution within the screw channel is studied in detail. It is found that the pressure developed at the die is strongly affected by the throughput, the reaction rate $S$ and the power law index.

\section{Problem formulation}

The simplified geometry of a single-screw extruder and the cross section of a screw channel are shown in figure 1. For ease of visualization and analysis, the coordinate system is fixed to the screw root and, thus, the barrel moves in a direction opposite to the screw rotation. Such a formulation is commonly employed in the literature (Fenner 1979; Tadmor \& Gogos 1979).

The following assumptions are made in deriving the governing equations from the basic conservation principles.

(1) Curvature of the screw channel is small, enabling the channel helix to be "opened out" to obtain a simpler geometry for ease of computation. 


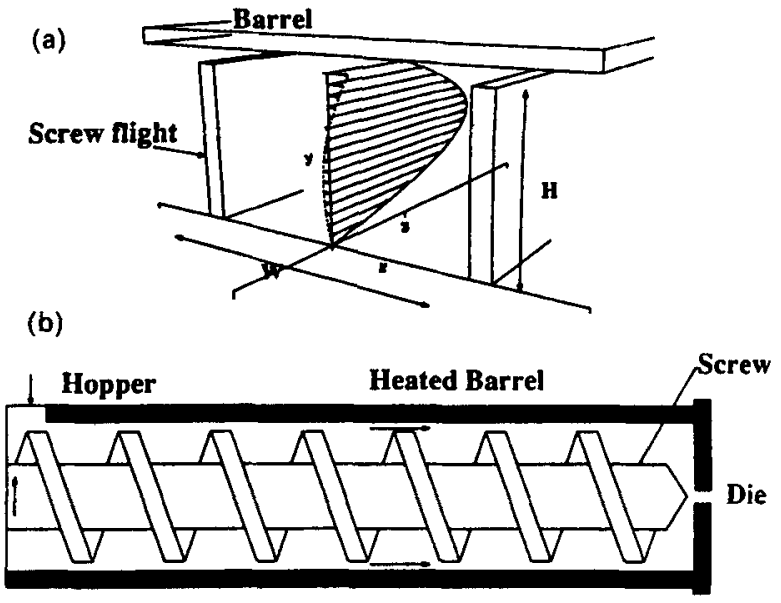

Figure 1. Simplified geometry of a single-screw extruder with a rectangular screw profile. (a) Perspective and (b) crosssectional views.

(2) The screw profile is rectangular, the width $(W)$ being much larger than the depth $(H)$. Other shapes such as a trapezoid can also be simulated in this scheme by using an "equivalent" height.

(3) The lubrication approximation is valid. For the highly viscous flow regime considered here for typical food materials, the Reynolds number is of the order of 0.0002 , showing that the inertial terms are indeed negligible in comparison to the viscous terms.

(4) Back flow in the channel cross-section is limited to a very small fraction of the flow rate. This assumption relates the presence of a die at the end of the channel to the flow characteristics within the extruder; this coupling is discussed in detail later.

(5) The clearance between the screw flight and the barrel is assumed to be enough to permit one to neglect the leakage across the flights from one screw channel to the neighbouring one.

(6) The diffusion of moisture into the food material and its absorption due to gelatinization is modelled as a zeroth-order reaction.

For steady, developing, two-dimensional flow of a homogeneous fluid in a singlescrew extruder with a shallow channel (i.e., for $H \ll W$ in figure 1), after neglecting the inertia terms (creeping flow approximation: Schlichting 1979) in the $x$ and $z$ directions, the equations for the conservation of momentum become:

$$
\begin{aligned}
& \frac{\partial p}{\partial x}=\frac{\partial\left(\tau_{y x}\right)}{\partial y}, \quad \frac{\partial p}{\partial y}=0, \quad \frac{\partial p}{\partial z}=\frac{\partial\left(\tau_{y z}\right)}{\partial y}, \\
& \tau_{y x}=\mu(\partial u / \partial y), \quad \tau_{y z}=\mu(\partial w / \partial y) .
\end{aligned}
$$

The energy equation, after employing the lubrication approximation, becomes (Kays \& Crawford 1980):

$$
\varrho C w \frac{\partial T}{\partial z}=\frac{\partial}{\partial y}\left(k \frac{\partial T}{\partial y}\right)+\tau_{y x} \frac{\partial u}{\partial y}+\tau_{y z} \frac{\partial w}{\partial y} .
$$

Mathematically speaking, the energy equation is parabolic in the $z$-direction. In actual practice, there is a strong restriction to the flow in the form of a die at the end of the extruder. The characteristics of the flow in the extruder are strongly coupled 
to that inside the die. For small throughputs, there is back flow in the extruder channel, and this makes the problem elliptic. In such circumstances, the fluid flow and the heat transfer at the die also need to be simulated and coupled to the extruder simulation. The present study is, however, restricted to the simulation of the transport phenomena in the extruder channel. The parabolic nature of the governing equation in the $z$-direction allows us to use a marching scheme, as described in the next section.

The constitutive equation for viscosity of starch materials such as Amioca and Hylon 7 is written as:

where

$$
\mu=\mu_{0}\left(\dot{\gamma} / \dot{\gamma}_{0}\right)^{(n-1)} \exp (b / T) \exp \left(-B_{m} C_{m}\right)
$$

$$
\dot{\gamma}=\left[(\partial u / \partial y)^{2}+(\partial w / \partial y)^{2}\right]^{1 / 2} .
$$

The dependence of viscosity on temperature and moistr re content described above has been confirmed by rheological experiments carried out by Kokini et al (1987).

The mass diffusion equation also needs to be considered in addition to the continuity, momentum, and energy equations for food systems. The particular food systems we are interested in examining are starches of various types. For example, some of the important effects of moisture transport are manifested in the form of gelatinization of the granular food material, which is fed into the hopper of the screw extruder. This reaction occurs between the starch granules and the water molecules. In this study, for the modelling of moisture diffusion, gelatinization is defined as the process by which water is bonded to the starch granules in the food material and thus becomes unavailable for diffusion. The diffusivity of bound moisture is reduced by several orders of magnitude, and the food material is said to undergo a form of transition. This transition affects the heat absorbed as the material undergoes chemical changes during its passage along the screw channel. The moisture diffuses, gets absorbed, and is convected along the flow direction. In this study, the transport equation for moisture is written to include the effect of gelatinization, and the chosen constitutive equation for viscosity reflects the experimentally observed dependence of viscosity on moisture concentration. It has been observed (Harper 1980) that even small changes in the moisture content can result in large changes in viscosity. The mass transfer equation is written as:

$$
w \frac{\partial c_{m}}{\partial z}=\frac{\partial^{2} c_{m}}{\partial y^{2}}+S^{\prime}\left(c_{m}\right)^{m}, \quad S^{\prime}=0 \quad \text { for } \quad T<T_{\mathrm{gel}} .
$$

The last term in the right-hand side of the equation represents a source/sink for the diffusing species due to reaction. This term becomes operative only when the temperature exceeds the temperature needed for the onset of the gelatinization reaction, $T_{\text {gel }}$. A zero-order reaction is considered in this study, with $m=\mathrm{C}$. Wang et al (1989) have examined the rates of conversion of starch materials based on shear and thermal processing history. The proportionality constant is a function of the temperature and the shear rate, but is taken as constant in this study as a first approximation, and a parametric study is carried out.

The boundary conditions are prescribed as shown in figure 2 . The screw has been taken as isothermal, at $T_{b}$, in most studies reported in the literature. However, a more practical circumstance is represented by the adiabatic condition at the screw surface (Karwe \& Jaluria 1990). Otherwise, a conjugate problem needs to be examined, where the conduction in the screw is coupled to the conduction and convection inside the flow channel.

Two constraints arise on the basis of flow rate conservation considerations. If the 
Fully Developed Isothermal Flow at the Inlet
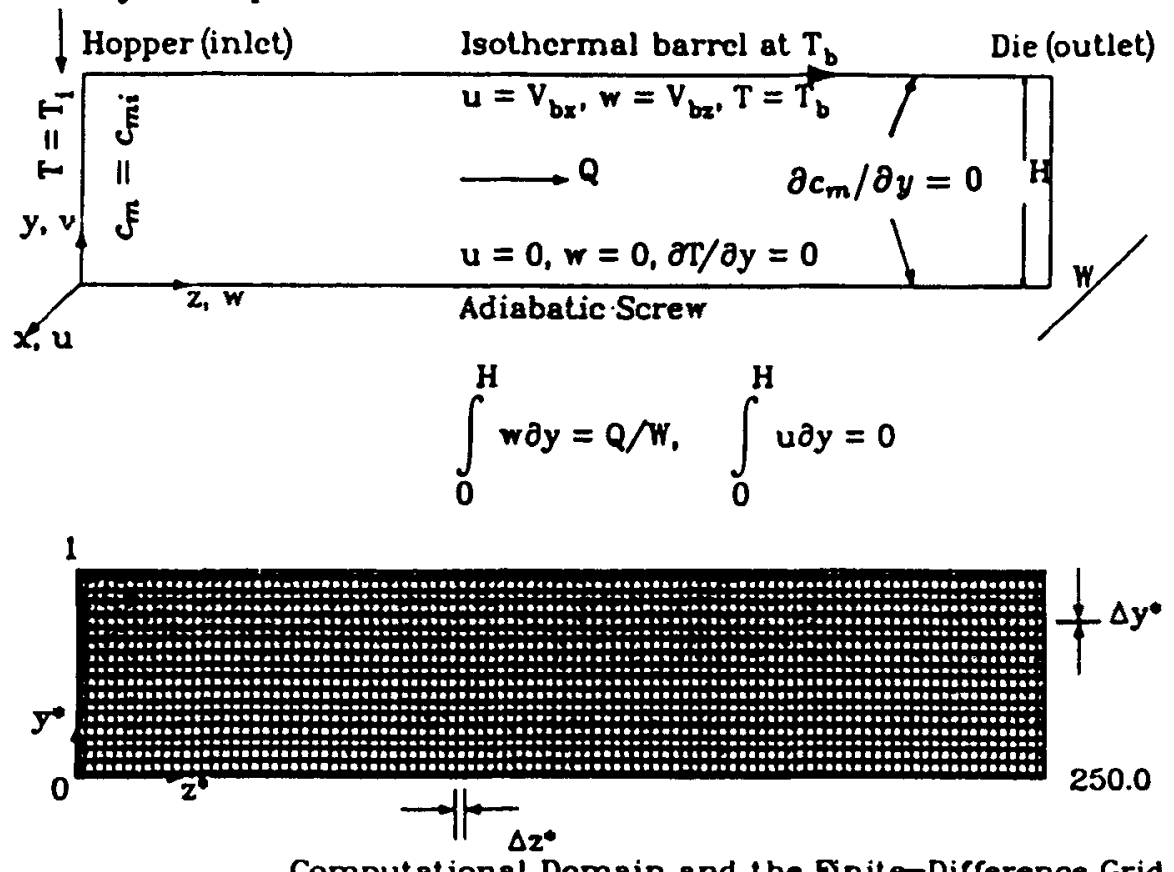

Figure 2. Boundary conditions for the numerical model, employing a coordinate system fixed to the screw.

leakage flow across the screw flights is taken as negligible, these constraints are given, for a total volumetric flow rate $Q$, by

$$
\int_{0}^{H} u \mathrm{~d} y=0, \text { and } \int_{0}^{H} w \mathrm{~d} y=Q / W .
$$

For tapered channels, the linear variation of channel height with distance along the screw helix can be written as:

$$
H(z)=H_{1}-\left(z / z_{\max }\right)\left(H_{1}-H_{2}\right) .
$$

The governing equations are nondimensionalized in terms of the following dimensionless variables:

$$
\begin{aligned}
x^{*} & =x / H_{2}, y^{*}=y / H, z^{*}=z / H_{2}, \\
u^{*} & =u / V_{b z}, w^{*}=w / V_{b z}, \\
\theta & =\left(T-T_{i}\right) /\left(T_{b}-T_{i}\right), c^{*}=c_{m} / c_{m i}, \\
\beta & =T_{b} / T_{i}, \beta_{1}=b / T_{i}, b_{m}=B_{m} c_{m i}, \\
\dot{\gamma}^{*} & =\dot{\gamma} H / V_{b z}, \bar{\mu}=\mu_{0}\left[\left(V_{b z} / H_{2}\right) / \dot{\gamma}_{0}\right]^{n-1}, \\
p^{*} & =p / \bar{p}, \bar{p}=\bar{\mu}\left(V_{b z} / H_{2}\right), V_{b z}=\pi D N(\cos \phi / 60), \\
\mathrm{Pe} & =V_{b z} H_{2} / \alpha, \mathrm{G}=\bar{\mu} V_{b z}^{2} / k\left(T_{b}-T_{i}\right), \\
\mathrm{Le} & =D / \alpha, S=S^{\prime} H_{2}^{2} c_{m i}^{m-1} / \alpha .
\end{aligned}
$$


The resulting dimensionless equations are:

$$
\begin{aligned}
& \frac{\partial p^{*}}{\partial x^{*}}=\left(\frac{H_{2}}{H}\right)^{n+1} \frac{\partial}{\partial y^{*}}\left\{\frac{\partial u^{*}}{\partial y^{*}}\left[\dot{\gamma}^{*}\right]^{(n-1)} \exp \left[\frac{\beta_{1}}{\theta(\beta-1)+1}\right] \exp \left(-b_{m} c^{*}\right)\right\} \\
& \frac{\partial p^{*}}{\partial z^{*}}=\left(\frac{H_{2}}{H}\right)^{n+1} \frac{\partial}{\partial y^{*}}\left\{\frac{\partial w^{*}}{\partial y^{*}}\left[\dot{\gamma}^{*}\right]^{(n-1)} \exp \left[\frac{\beta_{1}}{\theta(\beta-1)+1}\right] \exp \left(-b_{m} c^{*}\right)\right\} \\
& \operatorname{Pe} w^{*} \frac{\partial \theta}{\partial z^{*}}=\left(\frac{H_{2}}{H}\right)^{2} \frac{\partial^{2} \theta}{\partial y^{* 2}} \\
& +\left(\frac{H_{2}}{H}\right)^{n+1} \mathrm{G}\left[\dot{\gamma}^{*}\right]^{(n+1)} \exp \left[\frac{\beta_{1}}{\theta(\beta-1)+1}\right] \exp \left(-b_{m} c^{*}\right) \\
& \operatorname{Pe} w^{*} \frac{\partial c^{*}}{\partial z^{*}}=\operatorname{Le}\left(\frac{H_{2}}{H}\right)^{2} \frac{\partial^{2} c^{*}}{\partial^{2} y^{*}}+S c^{* m}, \quad S=0 \text { for } \theta<\theta_{\mathrm{gel}} \text {. }
\end{aligned}
$$

Similarly, the boundary conditions are also obtained in the dimensionless form. The constraints on the flow are obtained, in dimensionless form, as follows:

$$
\begin{aligned}
& \int_{0}^{1} u^{*} \mathrm{~d} y^{*}=0 \\
& \int_{0}^{1} w^{*} \mathrm{~d} y^{*}=(Q / W) / H V_{b z}=q_{v} H_{2} / H
\end{aligned}
$$

where

$$
q_{v}=(Q / W) / H_{2} V_{b z}
$$

Thus, for a given screw configuration, the parameters that govern the problem are $\psi, n, \beta, \beta_{1}, b_{m}, \theta_{\mathrm{gel}}, \mathrm{Pe}, \mathrm{G}, \mathrm{Le}$, and $q_{v}$.

\section{Solution procedure}

The dimensionless equations are solved by means of finite difference techniques. The computations were carried out over $y \times z$ grid sizes of $41 \times 101,61 \times 101$, and $81 \times 101$. The results were essentially unchanged when the grid was refined to $81 \times 121$ from $61 \times 101$ and therefore, a $61 \times 101$ grid was selected. Since the energy and mass transport equations, (10) and (11), are parabolic in $z$, boundary conditions are necessary only at $z=0$ to allow marching in the $z$-direction and, thus, obtain the solution in the entire domain. The throughput parameter $q_{v}$ determines the limits of applicability of the marching scheme. As a rough estimate, a value of $q_{v}$ less than 0.2 resulted in significant back flow, for typical values of the other parameters, limiting the use of marching for the numerical solution.

A method similar to the one developed by Fenner (1979) is employed for solving the momentum equations, (8) and (9), at a given $z$ location iteratively.

In dimensionless form, the momentum equations in $z$ and $x$ directions are:

$$
\pi_{z}=\frac{\partial}{\partial z^{*}}\left(\dot{\gamma}^{* n-1} \exp \left[\frac{\beta_{1}}{\theta(\beta-1)+1}\right] \exp \left(-b_{m} c^{*}\right) \frac{\partial w^{*}}{\partial y^{*}}\left(\frac{H_{2}}{H}\right)^{n+1}\right)
$$




$$
\pi_{x}=\frac{\partial}{\partial y^{*}}\left(\dot{\gamma}^{* n-1} \exp \left[\frac{\beta_{1}}{\theta(\beta-1)+1}\right] \exp \left(-b_{m} c^{*}\right) \frac{\partial u^{*}}{\partial y^{*}}\left(\frac{H_{2}}{H}\right)^{n+1}\right),
$$

where $\pi_{z}=\partial p^{*} / \partial z^{*}$ and $\pi_{x}=\partial p^{*} / \partial x^{*}$.

Integrating (13) and (14) over $y^{*}$, we get:

$$
\begin{aligned}
& \pi_{z}\left(y^{*}-y_{0}^{*}\right)=\dot{\gamma}^{* n-1} \exp \left[\frac{\beta_{1}}{\theta(\beta-1)+1}\right] \exp \left(-b_{m} c^{*}\right) \frac{\partial w^{*}}{\partial y^{*}}\left(\frac{H_{2}}{H}\right)^{n+1} \\
& \pi_{x}\left(y^{*}-y_{1}^{*}\right)=\dot{\gamma}^{* n-1} \exp \left[\frac{\beta_{1}}{\theta(\beta-1)+1}\right] \exp \left(-b_{m} c^{*}\right) \frac{\partial u^{*}}{\partial y^{*}}\left(\frac{H_{2}}{H}\right)^{n+1}
\end{aligned}
$$

where $y_{0}^{*}$ and $y_{1}^{*}$ represent $y$-locations in the channel (from the screw root to the barrel) where the shear stresses are zero.

Rearranging, we have

where

$$
\begin{aligned}
& \partial w^{*} / \partial y^{*}=\pi_{z}\left(y^{*}-y_{0}^{*}\right) F\left(y^{*}\right), \\
& \partial u^{*} / \partial y^{*}=\pi_{x}\left(y^{*}-y_{1}^{*}\right) F\left(y^{*}\right),
\end{aligned}
$$

$$
F\left(y^{*}\right)=\dot{\gamma}^{* 1-n} \exp \left[\frac{\beta_{1}}{\theta(\beta-1)+1}\right] \exp \left(b_{m} c^{*}\right)\left(\frac{H}{H_{2}}\right)^{n+1} \text {. }
$$

Squaring and adding (17) and (18), we get:

$$
\begin{aligned}
\left(\dot{\gamma}^{*}\right)^{2} & =\left(\partial w^{*} / \partial y^{*}\right)^{2}+\left(\partial u^{*} / \partial y^{*}\right)^{2} \\
& =\left[\pi_{z}^{2}\left(y^{*}-y_{0}^{*}\right)^{2}+\pi_{x}^{2}\left(y^{*}-y_{1}^{*}\right)^{2}\right] F^{2}\left(y^{*}\right)
\end{aligned}
$$

Solving for $\dot{\gamma}^{*}$, we get:

$$
\begin{gathered}
\dot{\gamma}^{*}=\left[\pi_{x}^{2}\left(y^{*}-y_{1}^{*}\right)^{2}+\pi_{z}^{2}\left(y^{*}-y_{0}^{* 2}\right]^{1 / 2 n} \times\right. \\
\exp \left[\frac{-\beta_{1} / n}{\theta(\beta-1)+1}\right] \exp \left(\frac{b_{m} c^{*}}{n}\right)\left(\frac{H}{H_{2}}\right)^{(n+1) / n}
\end{gathered}
$$

Substituting back into (19), we write

$$
\begin{gathered}
F\left(y^{*}\right)=\left[\pi_{z}^{2}\left(y^{*}-y_{0}^{*}\right)^{2}+\pi_{x}^{2}\left(y^{*}-y_{1}^{*}\right)^{2}\right]^{(1-n) / 2 n} \\
\exp \left[\frac{-\beta_{1} / n}{\theta(\beta-1)+1}\right] \exp \left(\frac{b_{m} c^{*}}{n}\right)\left(\frac{H}{H_{2}}\right)^{(n+1) / n}
\end{gathered}
$$

The velocities are obtained by integrating (17) and (18):

$$
\begin{aligned}
& w^{*}=\int_{0}^{y^{*}} \pi_{z}\left(\alpha-y_{0}^{*}\right) F(\alpha) \mathrm{d} \alpha, \\
& u^{*}=\int_{0}^{y^{*}} \pi_{x}\left(\alpha-y_{1}^{*}\right) F(\alpha) \mathrm{d} \alpha .
\end{aligned}
$$


The conditions to be satisfied by the velocities $u^{*}$ and $w^{*}$ are:

$$
\begin{aligned}
& w^{*}\left\{\text { at } y^{*}=1\right\}=1, \\
& u^{*}\left\{\text { at } y^{*}=1\right\}=\tan \phi, \\
& q_{v} H_{2} / H=\int_{0}^{1} w^{*} \mathrm{~d} y^{*}, \\
& 0=\int_{0}^{1} u^{*} \mathrm{~d} y^{*}
\end{aligned}
$$

After some algebraic manipulations, the following equations for the four unknowns in the left hand side are obtained:

where

$$
\begin{aligned}
\pi_{z} & =\left(J_{0}-J_{1}-J_{0} q_{v} H_{2} / H\right) /\left(J_{0} J_{2}-J_{1}^{2}\right), \\
\pi_{z} y_{0}^{*} & =\left(J_{1}-J_{2}-J_{1} q_{v} H_{2} / H\right) /\left(J_{0} J_{2}-J_{1}^{2}\right), \\
\pi_{x} & =\left(J_{0}-J_{1}\right) \tan \phi /\left(J_{0} J_{2}-J_{1}^{2}\right), \\
\pi_{x} y_{1}^{*} & =\left(J_{1}-J_{2}\right) \tan \phi /\left(J_{0} J_{2}-J_{1}^{2}\right),
\end{aligned}
$$

$$
J_{m}=\int_{0}^{1} \alpha^{m} F(\alpha) \mathrm{d} \alpha .
$$

The solution algorithm is enumerated below.

(1) Guess $\pi_{z}, \pi_{z} y_{0}^{*}, \pi_{x}, \pi_{x} y_{1}^{*}$.

(2) Calculate $F\left(y^{*}\right)$ using (22).

(3) Calculate the $J$ integrals using (27).

(4) Solve (26) using the Gauss-Jordan algorithm.

(5) Obtain converged flow solution at each time step.

(6) Using the converged flow solution, march down the channel to obtain temperature and moisture concentration fields at the next downstream location using (10) and (11).

(7) Go back to step 1 with the new set of temperature and concentration values.

The iterations in the flow calculation are complete when the pressure gradients satisfy the following convergence criterion:

$$
\max \left[\Delta\left(\partial p^{*} / \partial z^{*}\right), \quad \Delta\left(\partial p^{*} / \partial x^{*}\right)\right] \leqslant 10^{-4},
$$

where $\Delta$ stands for the absolute value of the fractional change between two consecutive iterations. This particular convergence criterion is not useful when the values of the pressure gradients become very small (i.e., close to zero). Under such circumstances, only the absolute change in the values of the pressure gradient is considered for convergence. Values of the criterion other than $10^{-4}$ were also tried, and it was found that satisfactory convergence was obtained with the foregoing value within a reasonable number of iterations (typically 5-10).

Using the boundary conditions, in terms of $u^{*}, w^{*}, \theta$, and $c^{*}$ at any upstream $z$ location, the energy equation (10), is solved to obtain the temperature distribution at the next downstream $z$ location. Equation $(10)$ is solved using the fully implicit scheme (Jaluria \& Torrance 1986). In this scheme, a tridiagonal system of equations 
is obtained by using the new, uncalculated, value of the dependent variable from the differencing operation in the $y$-direction at any nodal point in the numerical scheme. The tridiagonal system is solved using the well-known Thomas (TDMA) algorithm, which is very efficient (Jaluria 1988). The mass transport equation, (11), is solved next using, once again, the fully implicit scheme to obtain the values of concentration at the next location in the marching direction. With the temperature and concentration distributions obtained at the next downstream location, the momentum equations, (8) and (9), are solved iteratively as discussed above to obtain the velocity distribution there. This procedure is repeated until the end of the extruder channel is reached. The integration in (27) was carried out numerically using Simpson's one-third rule (Jaluria 1988).

During the course of carrying out the parametric study, a number of interesting features of the numerical scheme were noted. It was found that viscosity characterization in terms of $\beta, \beta_{1}$, and $b_{m}$ is very closely linked to the magnitude of the gelatinization rate $S$ that is specified for the particular reaction. For given values of $\beta, \beta_{1}$, and $b_{m}$, the pressure development as a function of downstream channel distance is affected considerably by minor changes in $S$. In addition, the range of $q_{v}$ that produces reasonable pressure development with channel distance is quite limited by back flow considerations on the one hand and unacceptable pressure drops on the other.

\section{Results and discussion}

Results are presented in terms of distributions of velocity, temperature, and moisture concentration along the screw channel as well as across the channel depth. The variation of pressure along the extruder channel as a function of the governing parameters is a useful measure of its pumping ability, and is examined here. Contour plots of constant velocity, temperature, and moisture are also obtained. The coordinate system is fixed to the screw as described earlier. For ease of discussion and presentation of results, some of the parameters are kept constant while the others are changed parametrically to study their effect on the transport processes. For the results presented here, the following are kept constant: $\mathrm{Pe}=7050, \mathrm{Le}=0.001, \theta_{\mathrm{gel}}=0.5, \phi=16.54^{\circ}$, $\psi=0.0^{\circ}$, and $\beta=1 \cdot 134$. In addition, the calculations are carried out for $\beta_{1}=10$ and $b_{m}=0$. Other values of these parameters were also considered, but those results are not reported here for brevity.

Figure 3 shows the calculated isotherms and the temperature profiles at four downstream locations for the nontapered case for Amioca, $n=0 \cdot 3$. The parameter $\mathrm{G}$, which represents the relative importance of viscous dissipation compared to heat conduction in the $y$-direction is quite high and gives rise to fluid temperatures that are higher than the barrel temperature (i.e. $\theta>1$ ). Consequently, heat transfer occurs from the fluid to the barrel, which may, therefore, have to be cooled in the sections close to the die to ensure that it is maintained at a particular temperature. For smaller values of $G$, the more common situation of heat transfer from the barrel to the fluid arises. The temperature gradient, $\partial T / \partial y$, at the barrel is also higher than that at the screw root because the heat generated by viscous dissipation has to be conducted away from the barrel to the ambient, whereas the screw root is adiabatic. Material temperatures are typically seen to be higher than imposed barrel temperatures, and this has important implications in extruder design.

Figure 4 shows the moisture concentration contours. The effect of the sink term 

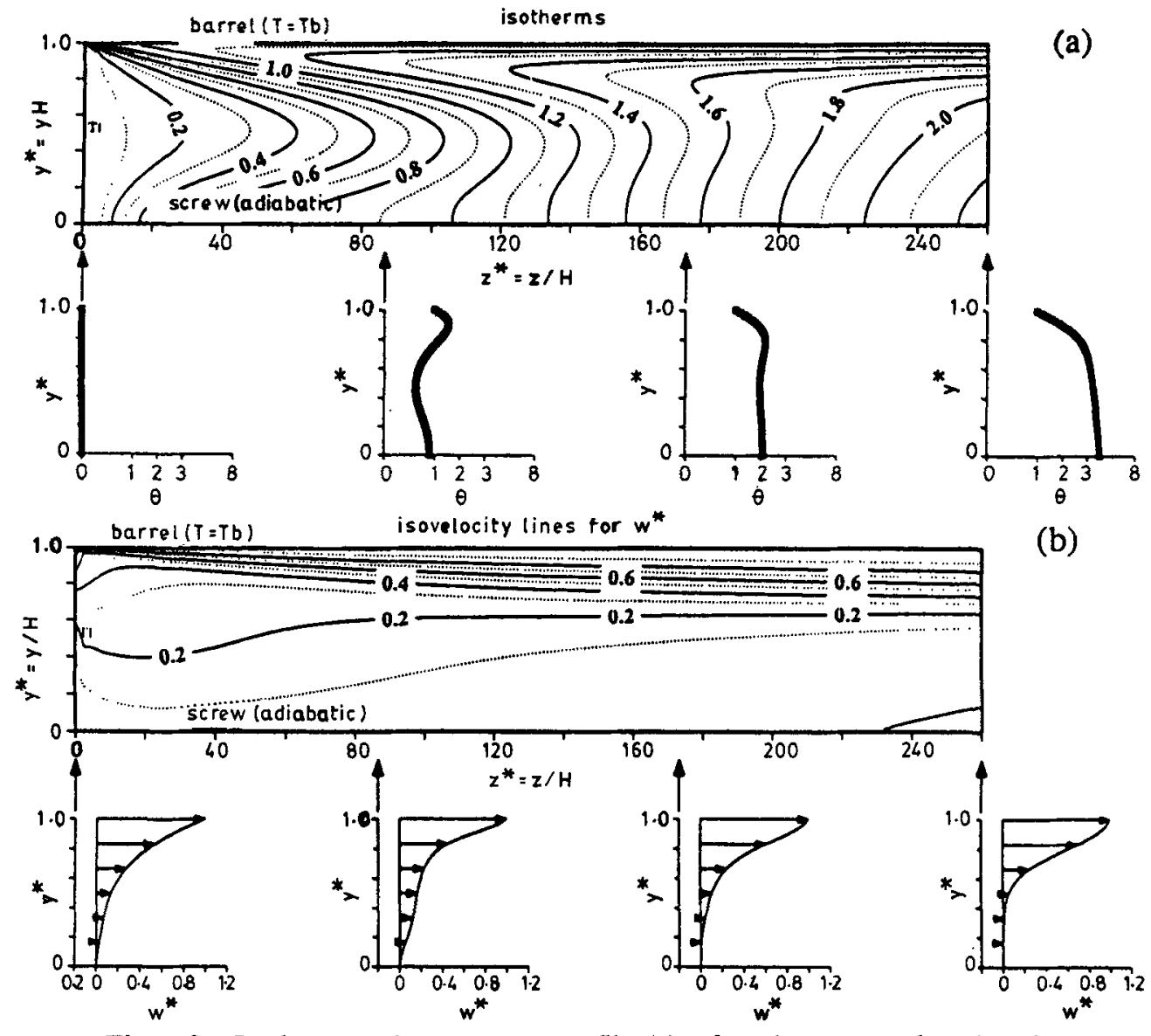

Figure 3. Isotherms and temperature profiles (a) at four downstream locations for Amioca with $n=0.3, \phi=16.54^{\circ}, q_{v}=0.25, \mathrm{Pe}=7050, \mathrm{G}=0.005, \beta=1.134$, $\beta_{1}=10.0, b_{m}=1 \cdot 0, S=-1000, \mathrm{Le}=0.001, \theta_{\mathrm{gel}}=0.5$, no taper. Also shown is the velocity field (b).
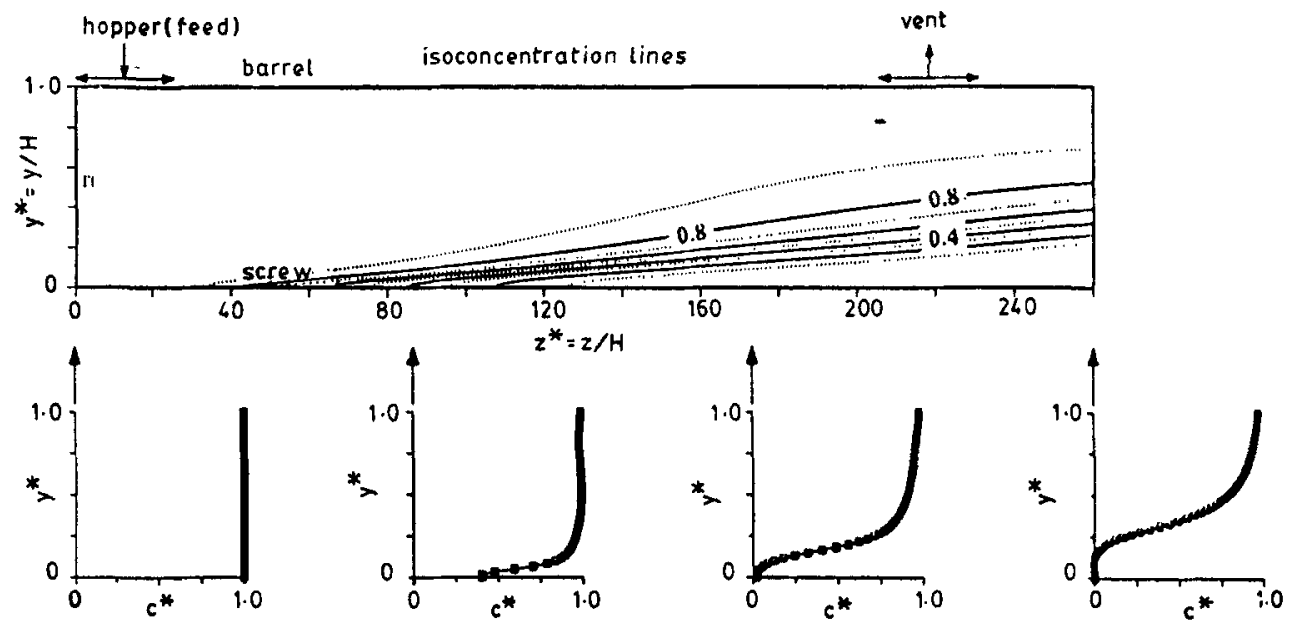

Figure 4. Lines of constant moisture concentration $c^{*}$, along with the corresponding profiles, at four downstream locations for Amioca for the conditions given in figure 3. 

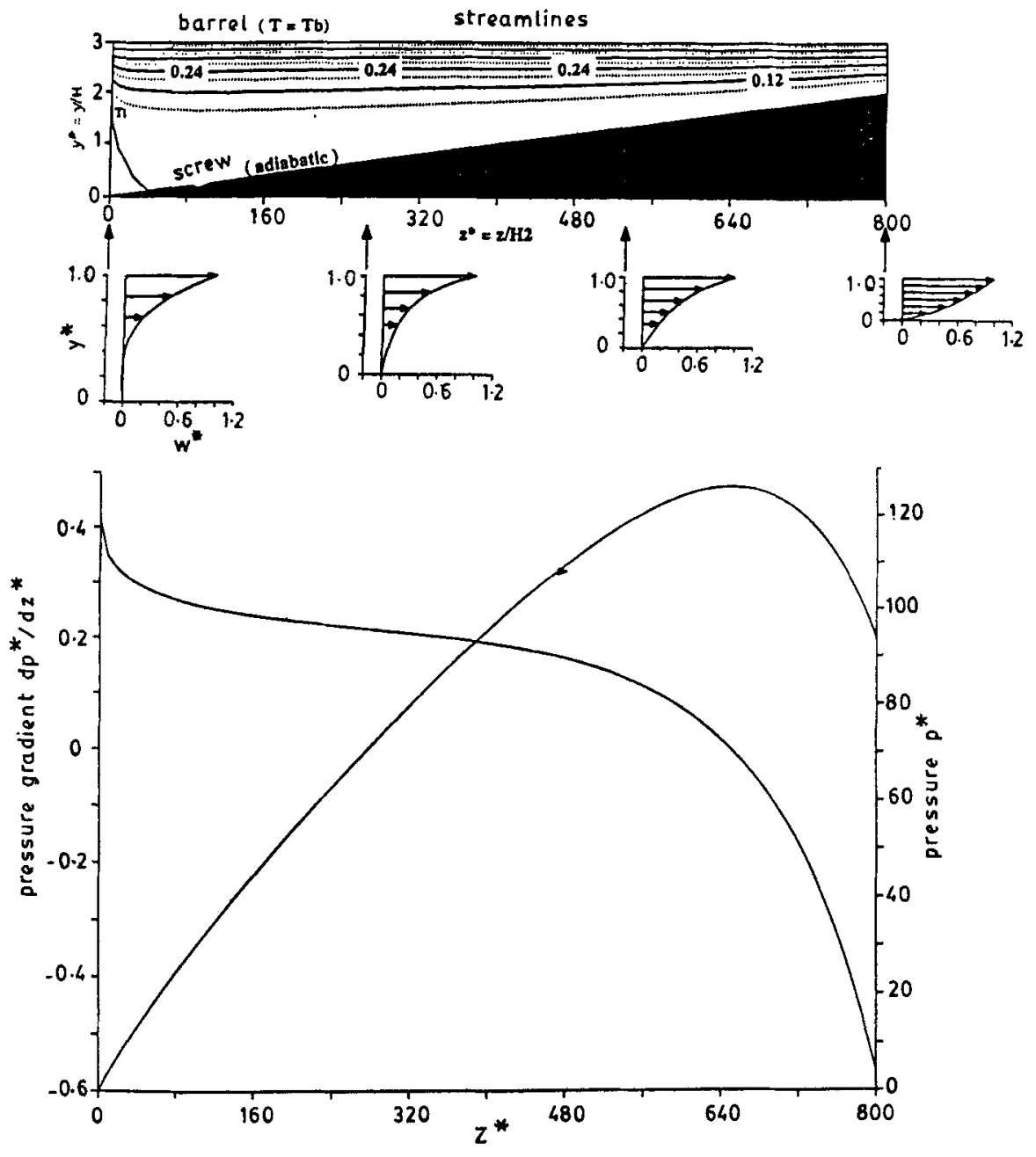

Figure 5. Streamlines and velocity profiles at four downstream locations, and pressure and pressure gradient variation with $z^{*}$ for Hylon 7 in a tapered channel with $n=0.5, q_{v}=0.2, \mathrm{Pe}=3000, \mathrm{G}=1 \cdot 0, \beta=1 \cdot 134, \quad \beta_{1}=5 \cdot 0, b_{m}=0.0$, and $\psi=0.1432^{\circ}$.

$S$, due to the reaction, is manifested in the form of a decrease in the moisture concentration due to bonding of water for gel formation as the material reaches the gelatinization temperature $\theta_{\text {gel. }}$. This loss of moisture occurs at a rate specified by the sink term $S$ and is obviously more rapid for larger $S$. As the sink term is increased in magnitude from $S=0$ (i.e. no reaction), the sharp decrease in the moisture concentration occurs earlier along the screw channel as expected, and also occurs first at the screw root. The two variables that control the magnitude of viscosity that is, the temperature and the moisture concentration - act in opposing directions as the material is heated and thus loses moisture as a result of gelatinization. The temperature profiles indicate that the screw root gets hotter than the barrel wall for the set of conditions shown in figure 4 , and this leads directly to gelatinization near the screw first. However, a reaction rate that is a function of both temperature and shear is a more realistic representation of the cooking process. In such cases, the 
gelatinization contours may be quite different from the ones shown here. Effort is currently underway to incorporate thermal and shear effects into the model.

The results of the simulation for a typical taper angle of $0.1432^{\circ}$ are shown in figure 5. This taper angle corresponds to the Brabender single-screw extruder. The screw root is continuously tapered from the feed section to the die. The normalization for all lengths is based on the final channel depth $\left(\mathrm{H}_{2}\right)$ except for the vertical screw channel distance, $y$, which is scaled with the local depth $(H)$. In figure 5 , the taper section is enlarged for clarity.

Consider the case of the isothermal flow of a Newtonian fluid in a rectangular, tapered channel. Depending on the imposed pressures at either end of the channel, there could be a maximum in the pressure profile somewhere in between. This can be shown analytically. There is pure drag flow at the location of this maximum. For non-Newtonian flow, however, it is not clear whether this maximum necessarily should occur inside the extruder or outside it.

For the nonisothermal case, as the material flows in a tapered channel, it gets squeezed in the decreasing gap. However, the net flow rate has to be maintained at the same value at any channel cross section. As shown in figure 5, the spacing of the streamlines is closer toward the die. The velocity profiles at the four downstream locations show markedly different behaviour for tapered channels compared to the case with no taper. It may be noted that the initially curved velocity profile becomes linear (corresponding to drag flow) as the section becomes shallower. At the die section, the profile shows velocities in the channel that are larger than the barrel velocity (or the screw tip speed, in the screw-moving formulation), again demonstrating the squeezing effect. The temperature profile becomes almost completely uniform across the channel depth in the regions close to the die for a tapered channel. This temperature is also equal to the imposed barrel temperature. Compared to the case with no taper, this shows that the narrower gap results in better thermal mixing and uniformity. These results can be used advantageously in extruder design for situations in which a specific pressure level needs to be developed while maintaining good product mixing facilitated by the squeezing effect of the tapered channel.

Figure 6 shows the effect of varying the strength of the sink on the pressure rise along the channel. As compared to the case of $S=0$, the dimensionless pressure at the die is seen to be lower for higher sink strengths. The variation of the downstream

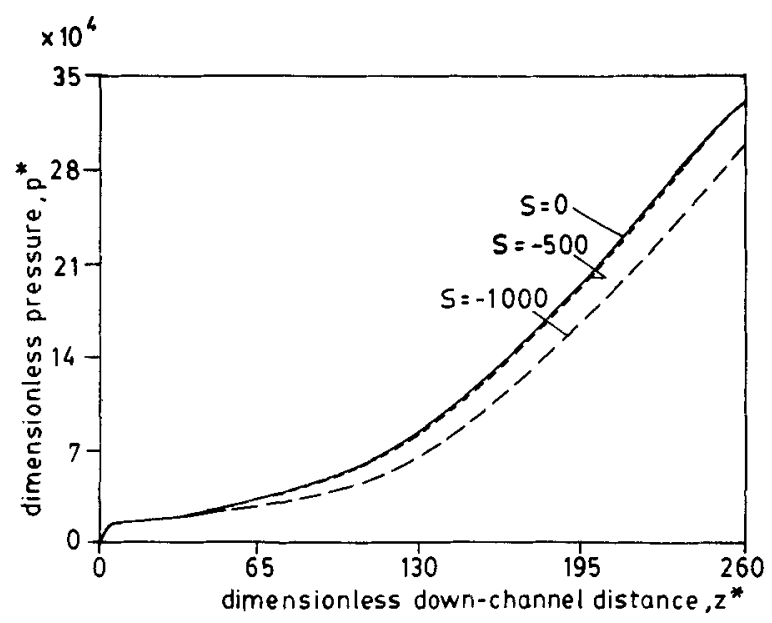

Figure 6. Effect of the strength of the moisture sink $S$ on the variation of the dimensionless pressure $p^{*}$ along the screw channel length $z^{*}$ for the conditions given in figure 3. 


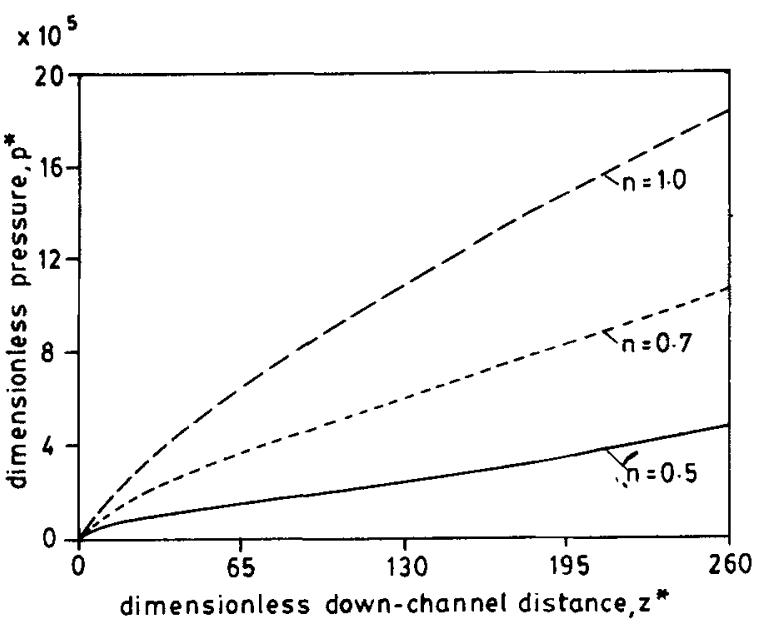

Figure 7. Effect of the power law index $n$ on the variation of dimensionless pressure $p^{*}$ along the screw channel length $z^{*}$ for $q_{v}=0 \cdot 3, \mathrm{G}=0 \cdot 001$, and other conditions as in figure 3 .

pressure gradient (not shown here) with the channel length shows that there is a kink in the profile at the point where the gelatinization first occurs. However, this rise in the value of $\mathrm{d} p / \mathrm{d} z$ is not strong enough to counter the temperature effect, which tends to lower the viscosity and, thus, makes the material flow more easily.

Figure 7 shows the effect on the pressure obtained in the extruder channel of changing the power law index $n$ of the material. Starches can be typically characterized by power law indices of less than $0 \cdot 5$. The curves are for $\mathrm{G}=0.001, q_{v}=0.3$, and $S=-1000$. Thus, only $n$ is varied, keeping the rest of the terms in (4) the same. This implies a higher viscosity for higher $n$ at a given shear rate. These results indicate the effect of $n$, though no actual physical circumstance is simulated here. The pressure rises from the hopper to the die along the serew helix, as expected. The pressure gradient is higher for increasing $n$. For non-Newtonian fluids, $n<1$, and the viscosity decreases with an increase in shear rate. The Newtonian fluid, $n=1$, therefore, gives rise to larger viscous drag, which in turn means that larger pressure gradients are required to overcome it.

The effect of the throughput $q_{v}$ on the pressure development at the die is investigated next. As shown in figure 8 , a smaller value of $q_{v}$, which corresponds to greater

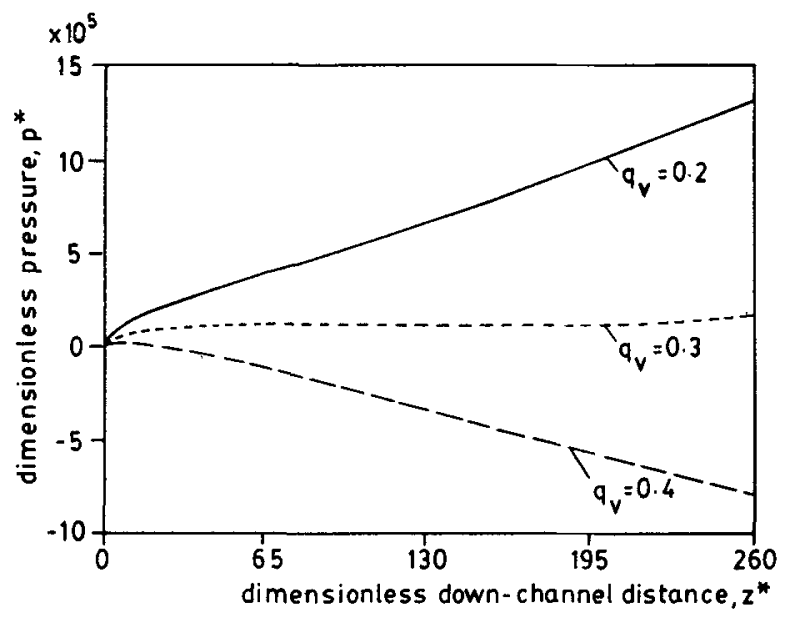

Figure 8. Effect of the throughput $q_{v}$ on the variation of dimensionless pressure $p^{*}$ along the screw channel length $z^{*}$ for $n=0.5, \quad \mathrm{G}=0.0$, and other conditions as in figure 3. 
restriction to the flow at the die, results in a larger pressure rise as compared to the case with $q_{v}=0.4$, which is close to the open die situation for the isothermal flow of Newtonian fluids, $q_{v}=0.5$. For the particular set of conditions here, a larger value of $q_{v}$ results is pressure loss from the hopper to the die. This is because the balance between the pressure buildup as the material flows through the extruder channel, and the easing of pressure due to the decrease of viscosity is not maintained. If the flow rate is increased, the pressure at the die decreases further. Therefore, there is a limiting value of the throughput $q_{v}$ that can be fed through an extruder beyond which pressure development and, correspondingly, the development of desirable material quality, are affected considerably. This limiting value depends on the particular conditions governing the flow: for example, the power law index $n$ and the viscosity coefficients $\beta, \beta_{1}$, and $b_{m}$. For isothermal, Newtonian flow, a simple theoretical analysis shows that $q_{v}=0.5$ corresponds to the throughput in the extruder in the absence of a die. For the nonisothermal, polymeric flow considered here, this value for the no-die situation is difficult to estimate, but is typically around 0.3 .

\section{Conclusions}

A numerical simulation of the transport phenomena occurring in the flow of food materials through a single-screw extruder has been carried out. For significant viscous dissipation within the material, the material temperature rises above the imposed barrel temperature by as much as $100 \%$. The flow field, however, is not strongly affected by this change. The moisture ooncentration contours provide a quantitative estimate of the extent of cooking that the food material undergoes. Moisture removal and bonding due to gelatinization of starch take place earlier and first at the screw root for higher reaction rate. Gelatinization increases the viscosity significantly, which in turn causes larger viscous heating and, consequently, cooking of the food material. Pressure development at the end of the extruder is significantly affected by the throughput. For throughputs larger than a limiting value, typically $0 \cdot 3$, the pressure can actually decrease from the hopper to the die, which means that the material has to be pumped through the extruder. Tapering of the screw channel is an important means of controlling the pressure development at the die.

This is publication No. F-10544-4-91 of the New Jersey Agricultural Experiment Station supported by State Funds and the Center for Advanced Food Technology (CAFT). The Center for Advanced Food Technology is a New Jersey Commission on Science and Technology Center. This work was also supported in part by the US Army Research Office. The authors would like to thank Dr M V Karwe and Professor V Sernas for discussions throughout this work.

\section{List of symbols}

$\begin{array}{ll}b & \text { temperature coefficient of viscosity, (4); } \\ b_{m} & =B_{m} c_{m i} ; \\ B_{m} & \text { moisture coefficient for viscosity; } \\ c_{m} & \text { moisture concentration; } \\ c_{m i} & \text { initial moisture concentration; }\end{array}$


$c^{*} \quad$ dimensionless $c_{m}$;

C specific heat of the fluid;

$D$ mass diffusivity of moisture into the bulk material;

$D_{b} \quad$ barrel diameter;

$\mathrm{G} \quad$ Griffith number, $\mathrm{G}=\bar{\mu} V_{b z}^{2} / k\left(T_{b}-T_{i}\right)$;

$H$ height of the screw channel, as a function of $z$;

$H_{1} \quad$ initial screw channel height;

$\mathrm{H}_{2} \quad$ final screw channel height;

$k \quad$ thermal conductivity of the fluid;

$L \quad$ axial screw length;

Le Lewis number, representing the relative magnitude of mass diffusivity to thermal diffusivity;

$n$

$N \quad$ screw speed (rpm); power law index, (4);

$p \quad$ pressure;

$\bar{p} \quad$ reference pressure, $\bar{p}=\bar{\mu} V_{b z} / H_{2}$;

$p^{*} \quad$ dimensionless pressure;

Pe Peclet number, $\mathrm{Pe}=\mathrm{V}_{b z} \mathrm{H}_{2} / \alpha$;

$q_{v} \quad$ dimensionless volumetric flow rate (throughput);

$Q$ total volumetric flow rate;

$S$ dimensionless sink term for moisture;

$s^{\prime} \quad$ rate of reaction;

$t \quad$ time;

$\bar{t} \quad$ average residence time;

$T$ temperature;

$T_{b} \quad$ barrel temperature;

$T_{i} \quad$ inlet temperature;

$u \quad$ velocity component in $x$-direction;

$v \quad$ velocity component in $y$-direction;

$V_{b} \quad$ tangential barrel velocity, $V_{b}=\pi D_{b} N / 60$;

$V_{b x} \quad$ component of $V_{b}$ along $x, V_{b x}=V_{b} \sin (\phi)$;

$V_{b z} \quad$ component of $V_{b}$ along $z, V_{b z}=V_{b} \cos (\phi)$;

$w \quad$ velocity component in $z$-direction;

$W \quad$ width of the screw channel;

$x \quad$ coordinate axis normal to screw flights; coordinate axis normal to the screw root; coordinate axis along the screw channel;

$\alpha \quad$ thermal diffusivity, $\alpha=k / \varrho C$;

$\beta \quad$ dimensionless $T_{b}, \beta=\mathrm{T}_{b} / T_{i}$;

$\beta_{1} \quad$ dimensionless $b, \beta_{1}=b / T_{i}$;

$\phi \quad$ screw helix angle;

$\dot{\gamma} \quad$ strain rate;

$\dot{\gamma}_{0} \quad$ reference strain rate, $(4)$;

$\dot{\gamma}^{*} \quad$ dimensionless strain rate;

$\mu_{0} \quad$ reference viscosity, (4);

$\mu \quad$ average viscosity, $\bar{\mu}=\mu_{0}\left[V_{b z} / H_{2} \dot{\gamma}_{0}\right]^{n-1}$;

$\psi \quad$ taper angle for a tapered screw channel;

$\theta$ dimensionless temperature, $\theta=\left(T-T_{i}\right) /\left(T_{b}-T_{i}\right)$

$Q \quad$ density of the fluid;

$\tau \quad$ shear stress. 


\section{Superscripts}

* dimensionless quantity.

\section{References}

Elbirli B, Lindt J T 1984 A note on numerical treatment of the thermally developing flow in screw extruders. Polym. Eng. Sci. 24: 482-487

Fenner R T 1977 Developments in the analysis of steady screw extrusion of polymers. Polymer 18: $617-622$

Fenner R T 1979 Principles of polymer processing (New York: Chemical Publishing)

Harper J 1980 Food extrusion (Boca Raton, FL: CRC Press)

Jaluria Y 1988 Computer methods for engineering (Needham Heights, MA: Allyn \& Bacon)

Jaluria Y, Torrance K E 1986 Computational heat transfer (New York: Hemisphere)

Karwe M V, Jaluria Y 1990 Numerical simulation of fluid flow and heat transfer in a single screw extruder for non-Newtonian fluids. Numer. Heat Transfer A17: 167-190

Kays W M, Crawford M E 1980 Convective heat and mass transfer 2nd edn (New York: McGraw-Hill)

Kokini J L 1987 Physical forces in food systems. Research accomplishment report, Center for Advanced Food Technology, Rutgers University, New Brunswick, New Jersey

Schlichting H 1979 Boundary layer theory (New York: McGraw-Hill)

Tadmor Z, Gogos C 1979 Principles of polymer processing (New York: Wiley)

Wang S S, Chiang C C, Yeh A I, Zhao B, Kim I H 1989 Kinetics of phase transition of waxy corn starch at extrusion temperatures and moisture contents. J. Food Sci. 54: $1298-1301$ 